\title{
Effect of microwave irradiation on reactions of 5-arylfuran-2-carboxaldehydes with some active methylene compounds
}

\author{
Estera Rábarová, ${ }^{a}$ Pavol Koiš, ${ }^{b}$ Margita Lácová, ${ }^{b}$ and Alžbeta Krutošíková* ${ }^{a}$ \\ ${ }^{a}$ Department of Chemistry, Faculty of Natural Sciences, University of St. Cyril and Methodius, \\ SK-917 01 Trnava, Slovak Republic \\ ${ }^{b}$ Department of Organic Chemistry, Faculty of Natural Sciences, Comenius University \\ SK-842 15 Bratislava, Slovak Republic \\ E-mail: krutosik@ucm.sk
}

(received 09 Mar 04; accepted 25 May 04; published on the web 06 Jun 04)

\begin{abstract}
The reactions of 5-arylfuran-2-carboxaldehydes with seven compounds containing an active methylene group have been studied in acetic anhydride, by both "classical" heating and under microwave assisted conditions. The compounds used are: ethyl 1,3-dioxo-indane-2-carboxylate, 2-amino-1-methyl-1,5-dihydro-4H-imidazol-4-one (creatinine), 2-thioxoimidazolidin-4-one (2thiohydantoin), 1,3-thiazolidine-2,4-dione, 2-(1H-benzimidazol-2-ylthio)acetic acid, hippuric acid and 4-phenyl-4-oxobutanoic acid. The beneficial effect of microwave irradiation on these condensations was found in a shortening of the reaction time and increase in the yields.
\end{abstract}

Keywords: 5-Arylfuran-2-carboxaldehydes, condensation reactions, microwave synthesis, structure, ${ }^{1} \mathrm{H}$ NMR spectra

\section{Introduction}

This paper is a continuation of our previous publications in which we have studied the beneficial effect of microwave irradiation on condensation reactions. ${ }^{1-4}$ The microwave-induced activation and acceleration of various reactions has several advantages and is suitable for use with polar and thermolabile compounds. ${ }^{5}$ The high irradiating efficiency in a short time gives rise to remarkable rate enhancement. We report herein the application of microwave irradiation to the condensation reaction between 5-arylfuran-2-carboxaldehydes (1) and the sodium salt of ethyl 1,3-dioxoindane-2-carboxylate (2), 2-amino-1-methyl-1,5-dihydro-4H-imidazol-4-one (creatinine) (3), 2-thioxoimidazolidin-4-one (2-thiohydantoin) (4), 1,3-thiazolidine-2,4-dione (5), 2-(benzimidazol-2-ylthio)acetic acid (6), Hippuric acid (7), and 4-phenyl-4-oxobutanoic acid (8). 
Both components used in the condensation reactions are important synthetic and biologically active compounds. For example, 5-arylfuran-2-carboxaldehydes (1) and some of their condensation products are utilized as bactericides, ${ }^{6}$ or antiviral compounds, ${ }^{7}$ and in addition have been found to have herbicidal, fungicidal and insecticidal activities. ${ }^{8,9}$

The active methylene components are known as intermediates or building blocks in organic synthesis; 3, 4, 7, especially, are used as precursors of amino acids by the Erlenmeyer-PlöchlBergmann synthesis.

The five-membered heterocycles 3-5 we have used exhibit high $\mathrm{CH}_{2}$ acidity and consequently these compounds can be condensed with aldehydes in the presence of a weak base. Condensation of $\mathbf{3}$ and $\mathbf{4}$ with aromatic aldehydes ${ }^{10,11}$ takes several hours at high temperature $\left(160-180{ }^{\circ} \mathrm{C}\right)$ under classical conditions. These compounds, without solvent, under focused microwave irradiation gave good yields of the products which were described by Villemin et al. ${ }^{12,13}$ We focused our attention on the condensation reaction of both components by the,"classical" procedure in order to optimize reaction conditions and yields, as well as on the comparison of the microwave irradiation effect.

\section{Results and Discussion}

For many years, the $\beta$-dicarbonyl compounds have been studied intensively owing to their synthetic and biological significance. The most known methods for preparation of $\beta$-dicarbonyl compounds are rearrangements of phthalides or the condensation of indane-1,3-dione with carbonyl compounds. ${ }^{14}$ Well known indane-1,3-dione derivatives are also important as anticoagulant drugs or rodenticides. ${ }^{15}$ Theoretical aspects and physicochemical properties of $\beta$ dicarbonyl compounds were studied systematically by Perjéssy et al. ${ }^{16,17}$

In this study we used a new, easy and effective modification of the preparation of indane-1,3dione derivatives, starting from the sodium salt of ethyl 1,3-dioxoindane-2-carboxylate (2), which is available via Dieckmann condensation ${ }^{18}$ of diethyl phthalate and ethyl acetate as a stable intermediate on the route to indane-1,3-dione.

The target 2-(5-aryl-2-furylmethylene)indane-1,3-diones (9a-c) were prepared from a mixture of equivalent amounts of aldehydes 1 and 2 (in 90\% yields) by heating (at $50-60^{\circ} \mathrm{C}$ ) and stirring (about 30-40 $\mathrm{min}$ ) in acetic anhydride without catalyst. The same mixture of 1 and 2 under microwave irradiation very rapidly afforded (2-4 min, at $250 \mathrm{~W}$ ) $85-90 \%$ yields of 9a-c (Scheme 1). We made an unsuccessful attempt at this condensation by heating components 1 and 2 in DMF as a solvent 
<smiles>[R]c1ccc(-c2ccc(C=O)o2)cc1</smiles>

1a-c

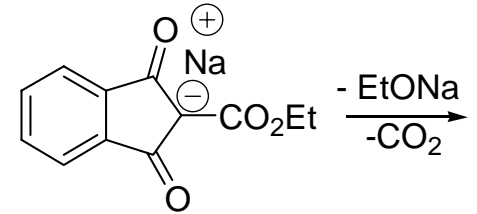

2

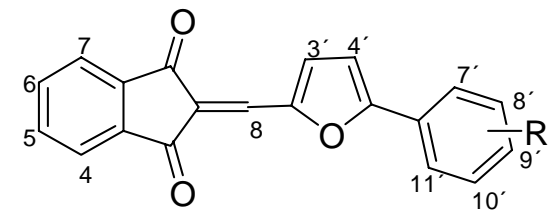

9a-c

$\mathrm{R}$ for: $\mathrm{a}=4-\mathrm{NO}_{2} ; \mathrm{b}=2-\mathrm{NO}_{2} ; \mathrm{c}=4-\mathrm{Br}$

\section{Scheme 1}

The continuation of this study was to investigate the condensation of five-membered lactams 3-5 with 1. The 5-arylfuran-2-carboxaldehydes (1) were condensed with three selected fivemembered heterocycles, 2-amino-1-methyl-1,5-dihydro-4H-imidazol-4-one (creatinine) (3), 2thioxoimidazolidin-4-one (2-thiohydantoin) (4), and 1,3-thiazolidine-2,4-dione (5), in acetic anhydride in the presence of potassium acetate under microwave or classical conditions afforded the condensation products 10-12 (Scheme 2).

The isolated products were formed from both components by the normal condensation. No secondary product from one molecule of aldehyde with two molecules of the methylene heterocycle was obtained.

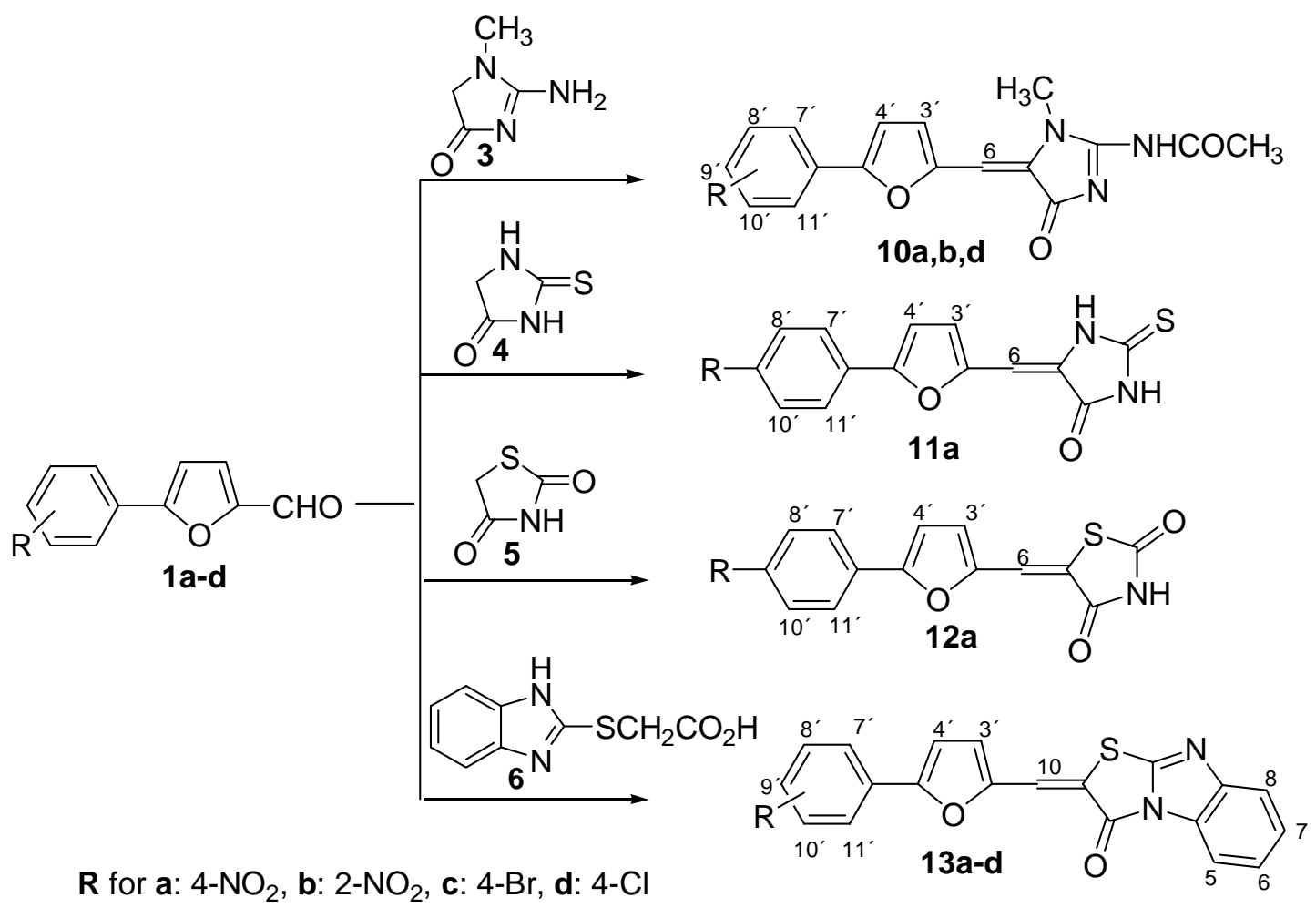

\section{Scheme 2}


The $\mathrm{NH}_{2}$ group of $\mathbf{1 0 a}, \mathbf{b}, \mathbf{d}$ was acetylated in the course of the reaction. The difference in reaction times between these two condensation methods-"classical" or the microwave procedure was evident. The duration of the reaction by the "classic" method was about $3-5 \mathrm{~h}$ at $100-110^{\circ} \mathrm{C}$, but under microwave conditions, 6-9 min. at $400 \mathrm{~W}$. The yields of the expected products by both procedures were comparable (Table 1). The reactions of 5-aryl-2-furancarboxaldehydes (1) with the acids 6-8 were realized between equimolar quantities of components, under irradiation or classical heating conditions, and produced condensation products in good yields (60-86\%) under both conditions, giving the compounds 13-15 (Schemes 2, 3, 4).

Table 1. Comparisons between "classical" and microwave procedures

\begin{tabular}{|c|c|c|c|c|}
\hline \multirow{2}{*}{ Compound } & \multicolumn{2}{|c|}{ Classical conditions } & \multicolumn{2}{|l|}{ MW conditions } \\
\hline & $\begin{array}{c}\text { Reaction time } \\
\qquad / \mathrm{h} .\end{array}$ & Yield \% & $\begin{array}{l}\text { Reaction time } \\
\text { min. }\end{array}$ & Yield \% \\
\hline 9a & 0.5 & 88 & 3 & 88 \\
\hline $9 b$ & 0.5 & 86 & 3 & 84 \\
\hline 9c & 0.6 & 92 & 4 & 90 \\
\hline 10a & 4 & 74 & 8 & 68 \\
\hline 10b & 4 & 68 & 8 & 67 \\
\hline 10d & 5 & 77 & 9 & 70 \\
\hline 11a & 3 & 65 & 6 & 69 \\
\hline $12 a$ & 3 & 54 & 6 & 66 \\
\hline $13 a$ & 4 & 80 & 9 & 78 \\
\hline 13b & 4 & 74 & 9 & 75 \\
\hline 13c & 5 & 82 & 10 & 79 \\
\hline 13d & 5 & 85 & 10 & 80 \\
\hline $14 a$ & 3 & 68 & 8 & 60 \\
\hline 14b & 4 & 70 & 10 & 66 \\
\hline $14 \mathrm{c}$ & 4 & 70 & 10 & 65 \\
\hline 14d & 2.5 & 54 & 7 & 50 \\
\hline $14 e$ & 3 & 68 & 8 & 60 \\
\hline $14 f$ & 3 & 72 & 8 & 60 \\
\hline $14 \mathrm{~g}$ & 3 & 72 & 8 & 62 \\
\hline $14 \mathrm{~h}$ & 4 & 78 & 11 & 68 \\
\hline 15a & 3 & 65 & 6 & 66 \\
\hline $15 b$ & 3 & 60 & 6 & 64 \\
\hline $15 c$ & 3 & 64 & 8 & 70 \\
\hline $15 d$ & 3 & 62 & 8 & 68 \\
\hline 15e & 3 & 71 & 7 & 67 \\
\hline
\end{tabular}




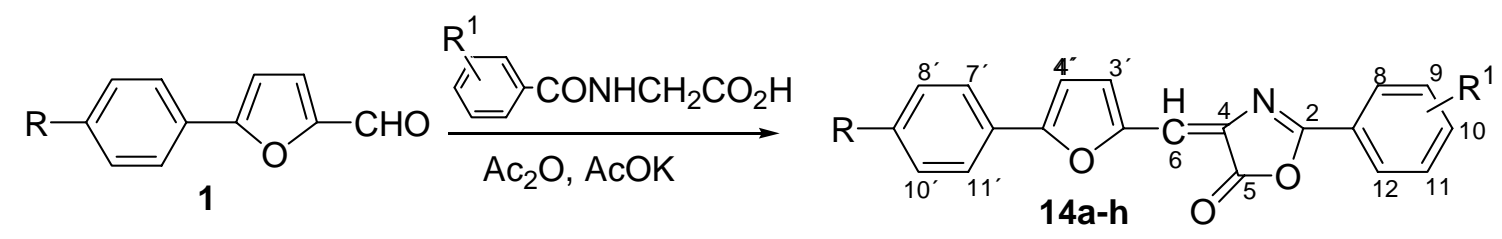

$\begin{array}{lcccccccc}\mathbf{1 4} & \mathbf{a} & \mathbf{b} & \mathbf{c} & \mathbf{d} & \mathbf{e} & \mathbf{f} & \mathbf{g} & \mathbf{h} \\ \mathbf{R} & \mathrm{NO}_{2} & \mathrm{Br} & \mathrm{Cl} & \mathrm{NO}_{2} & \mathrm{Br} & \mathrm{Cl} & \mathrm{Cl} & \mathrm{Cl} \\ \mathbf{R}^{1} & \mathrm{H} & \mathrm{H} & \mathrm{H} & 3-\mathrm{NO}_{2} & 3-\mathrm{NO}_{2} & 3-\mathrm{NO}_{2} & 4-\mathrm{NO}_{2} & \mathrm{NHCOCH}\end{array}$

\section{Scheme 3}

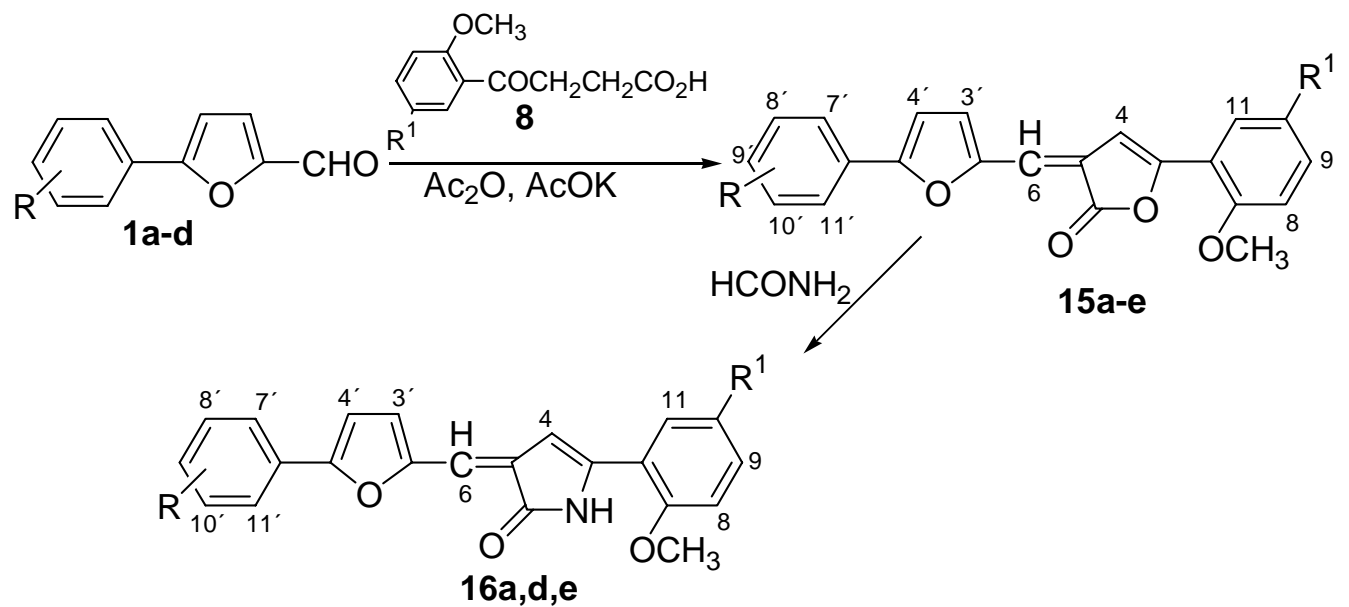

R for a: 4- $\mathrm{NO}_{2}$, b: 2-NO $\mathrm{NO}_{2}$ c: 4-Br, d and e: 4-Cl

$\mathbf{R}^{1}$ for a-d: $\mathrm{CH}_{3} \mathrm{O}$; for $\mathbf{e}: \mathrm{CH}_{3}$

\section{Scheme 4}

The corresponding substituted acetic acids 6-8 in acetic anhydride and base catalyst gave in situ the heterocycles A, B and C possessing an active methylene group, as illustrated in Scheme 5. The five-membered lactones and lactams A-C formed became the compounds with higher acidity of the active methylene group in comparison with the starting acids. Likewise, the cyclization of the substituted acetic acids 6-8 was carried out in acetic anhydride under microwave conditions for 6-10 min. smoothly and completely without by-products. 


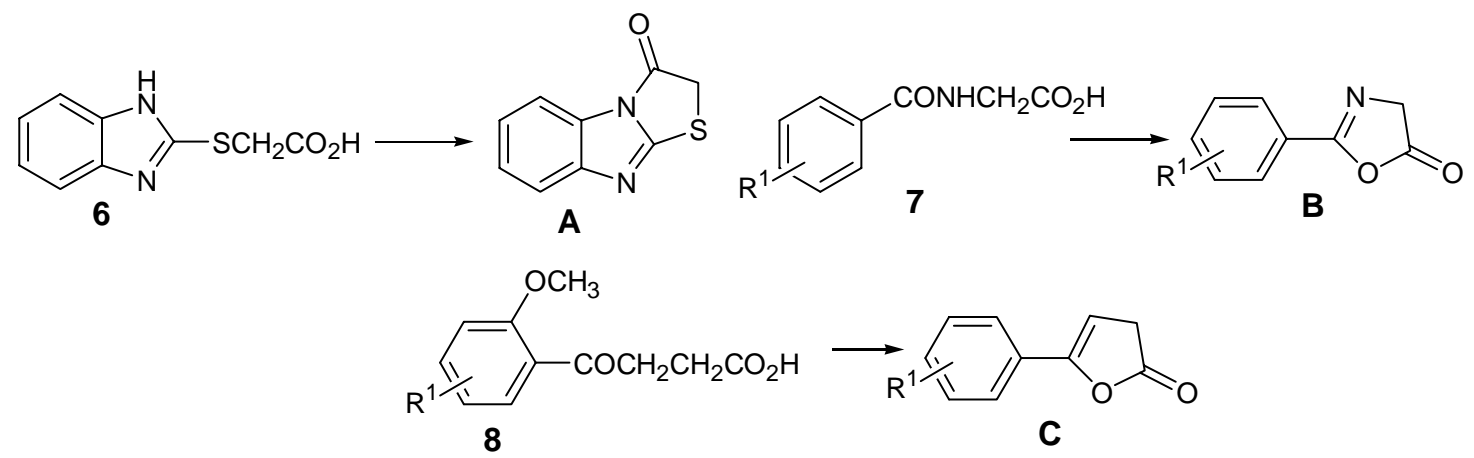

\section{Scheme 5}

The lactones 15, which are anhydrides of $\gamma$-hydroxy unsaturated acids, reacted with formamide at $110{ }^{\circ} \mathrm{C}$ forming lactams-derivatives of 2,3-dihydropyrrol-2-one 16 (Scheme 4), precursors for unsaturated $\gamma$-amino acids, which will be possible to obtain after hydrolysis. Formamide is a versatile reagent for inserting of amino group in molecules. Formamide reacts with $\alpha$-hydroxy-, $\alpha$-halo- or $\alpha$-aminoketones to form imidazole, also oxazoles can form into imidazoles, ${ }^{19}$ it changes phthalides into phthalimidines. ${ }^{20}$

The products of condensation are stable solid compounds, rather insoluble in common solvents, with high melting points. Isolation of products from the reaction mixture is very easy, because they are insoluble in acetic anhydride: after 1 day cooling in the refrigerator they were removed under suction and crystallized from ethanol, chloroform, or a mixture of ethanoldimethyl sulfoxide.

Because of their poor solubility in DMSO, we had to measure their ${ }^{1} \mathrm{H}-\mathrm{NMR}$ spectra at elevated temperature. The resonance signals and their multiplicity confirmed the proposed structures.

\section{Experimental Section}

General Procedures. Elemental analyses and ${ }^{1} \mathrm{H}-\mathrm{NMR}$ spectra characterized the products. Melting points of products were determined on Kofler hot-plate apparatus and are uncorrected. All solvents were pre-distilled and dried appropriately prior to use. ${ }^{1} \mathrm{H}-\mathrm{NMR}$ spectra were measured on a $300 \mathrm{MHz}$ spectrometer VARIAN GEMINI 200 in DMSO- $d_{6}$ or $\mathrm{CDCl}_{3}$ and with tetramethylsilane as internal standard. Coupling constants $(J)$ are quoted to the nearest $0.1 \mathrm{~Hz}$. Chemical shifts ( $\delta$-scale) are quoted in parts per million and the following abbreviations are used: $\mathrm{s}=$ singlet; $\mathrm{d}=$ =doublet; $\mathrm{t}=$ triplet; $\mathrm{q}=$ quartet; $\mathrm{m}=$ multiplet; $\mathrm{br}=$ broad. All microwave experiments were performed in a Lavis 1000 Milestone multi Quant (multimode) microwave oven. The apparatus was modified for laboratory applications equipped with magnetic stirring and an external reflux condenser. The temperature of mixture has been determined by reflux of acetic anhydride. Elemental analyses were determined using a Carlo Erba CHNS-OEA 1108- 
Elemental Analyser. The course of reaction in both methods was controlled by TLC chromatography (SILUFOL UV ${ }_{254}$ Kavalier, Votice, Czech Republic) in the system chloroformmethanol 9:1, visualizing spots with a UV lamp or iodine vapor. 5-Arylfuran-2-carboxaldehydes were prepared by the procedure in ref. 21, phenyl-4-oxobutanoic acid as in ref. 22 , and 2-(1Hbenzimidazolyl-2-thio)acetic acid as in ref. 23.

2-[5-(Aryl-2-furyl)methylene]indane-1,3-diones (9). (a) Classical method. To the stirring mixture of anhydrous acetic anhydride $(5 \mathrm{~mL})$ and 5-arylfuran-2-carboxaldehyde (1) (2 mmol), the sodium salt of ethyl 1,3-dioxoindane-2-carboxylate (2) was added in small portions during 20 min at $40{ }^{\circ} \mathrm{C}$. Stirring and heating was continued for a further $30 \mathrm{~min}$ at $50^{\circ} \mathrm{C}$ and then after cooling the reaction mixture was diluted with cold water $(20 \mathrm{~mL})$. The yellow precipitate was filtered off, dried, and crystallized from chloroform or ethanol.

(b) Method using microwave irradiation. The stirred mixture of the same composition as in the method above was irradiated in a microwave oven for 3-4 min at $250 \mathrm{~W}$. The work-up of the reaction mixture was identical with the "classical" method.

2-\{[5-(4-Nitrophenyl)-2-furyl)]methylene\}indane-1,3-dione (9a). Yield 88\%, m.p. 292-294 ${ }^{\circ} \mathrm{C}$. Anal. Calcd. for $\mathrm{C}_{20} \mathrm{H}_{11} \mathrm{NO}_{5}$ (345.3): C, 69.50; H, 3.19; N, 4.05. Found: C, 69.39; H, 3.01; N, $3.89 \% .{ }^{1} \mathrm{H}-\mathrm{NMR}\left(\mathrm{CDCl}_{3}\right) \delta_{\mathrm{H}} 7,17\left(\mathrm{~d}, 1 \mathrm{H},{ }^{3} \mathrm{~J}=3.9 \mathrm{~Hz}, \mathrm{H}-4^{\prime}\right), 7.73(\mathrm{~s}, 1 \mathrm{H}, \mathrm{H}-8), 7.75-7,85(\mathrm{~m}$, 2H, H-5,6), 7.97-8.05 (m, 4H, H-4, 7, 7', 11'), 8.33 (dd, $\left.1 \mathrm{H},{ }^{3} \mathrm{~J}=8.2 \mathrm{~Hz},{ }^{4} \mathrm{~J}=2 \mathrm{~Hz}, \mathrm{H}-8^{\prime}\right), 8.35$ (dd, $\left.1 \mathrm{H},{ }^{3} \mathrm{~J}=8.6 \mathrm{~Hz},{ }^{4} \mathrm{~J}=2 \mathrm{~Hz}, \mathrm{H}-10^{\prime}\right), 8.57$ (d, $\left.1 \mathrm{H},{ }^{3} \mathrm{~J}=3.8 \mathrm{~Hz}, \mathrm{H}-3^{\prime}\right)$.

2-\{[5-(2-Nitrophenyl)furyl]methylene\}indane-1,3-dione (9b). Yield 86\%, m.p. 246-248 . Anal. Calcd. for $\mathrm{C}_{20} \mathrm{H}_{11} \mathrm{NO}_{5}$ (345.3) C 69.50, H 3.19, N 4.05. Found: C, 69.46; H, 3.26; N, $3.99 \%$. ${ }^{1} \mathrm{H}$ NMR $\left(\mathrm{CDCl}_{3}\right) \delta_{\mathrm{H}} 6.98\left(\mathrm{~d}, 1 \mathrm{H},{ }^{3} \mathrm{~J}=3.7 \mathrm{~Hz}, \mathrm{H}-4\right), 7.51-7.59\left(\mathrm{~m}, 1 \mathrm{H}, \mathrm{H}-11^{\prime}\right), 7.69-$ 7.71 (m, 1H, H-9') 7.72 (s, 1H, H-8), 7.77-7.84 (m, 3H, H-5', 6, 10), 7.92 (dd, $1 \mathrm{H},{ }^{3} J=7.9 \mathrm{~Hz}$, $\left.{ }^{4} J=1.8 \mathrm{~Hz}, \mathrm{H}-8^{\prime}\right), 7.96-8.02$ (m, 2H, H-4,7), 8.61 (d, $\left.1 \mathrm{H},{ }^{3} \mathrm{~J}=3.7 \mathrm{~Hz}, \mathrm{H}-3{ }^{\prime}\right)$.

2-\{[5-(4-Bromophenyl)furyl]methylene\}indane-1,3-dione (9c). Yield 92\%, m.p. 243-244 ${ }^{\circ}$. Anal. Calcd. for $\mathrm{C}_{20} \mathrm{H}_{11} \mathrm{BrO}_{3}(379.2) \mathrm{C}, 63.35 ; \mathrm{H}, 2.92 ; \mathrm{Br}, 21.07$. Found: $\mathrm{C}, 63.55 ; \mathrm{H}, 2.88 ; \mathrm{Br}$, $21.24 \%$.

${ }^{1} \mathrm{H}-\mathrm{NMR}\left(\mathrm{DMSO}-d_{6}\right) \delta_{\mathrm{H}} 7,53\left(\mathrm{~d}, 1 \mathrm{H},{ }^{3} \mathrm{~J}=3.7 \mathrm{~Hz}, \mathrm{H}-4^{\prime}\right), 7.63(\mathrm{~s}, 1 \mathrm{H}, \mathrm{H}-8), 7.7-7,84(\mathrm{~m}, 4 \mathrm{H}, \mathrm{H}-$ $\left.5,6,7^{\prime}, 11^{\prime}\right), 7.9-8.1$ (m, 4H, H-4,7,8' $\left.10^{\prime}\right), 8.38$ (d, $\left.1 \mathrm{H},{ }^{3} \mathrm{~J}=3.8 \mathrm{~Hz}, \mathrm{H}-3^{\prime}\right)$.

\section{Synthesis of compounds 10-15}

(a) Classical method. A mixture of anhydrous acetic anhydride (5 mL), 5-arylfuran-2carboxaldehyde (1) (1.6 mmol), the active-methylene compound 3-8 (1,6 mmol) and freshly fused potassium acetate $(0.2 \mathrm{mmol})$ was slowly heated and stirred for three or five hours at $100-$ $120^{\circ} \mathrm{C}$; (the reaction mixture with compounds $\mathbf{1 0 , 1 1 , 1 2}$ was heating only at $60^{\circ} \mathrm{C}$ ). Then the reaction mixture was cooled, diluted with cold water and neutralized by solid potassium carbonate. The solid product was separated by vacuum filtration, dried, and crystallized from ethanol, chloroform or a mixture of DMSO-ethanol. 
(b) Method using a microwave irradiation. The same reaction mixture as above (but with only $3 \mathrm{~mL}$ of acetic anhydride) was stirred in the microwave oven at $400 \mathrm{~W}$ for $4-10 \mathrm{~min}$. The reaction mixture was worked up in a similar way to that given above.

2-Acetylamino-1-methyl-5-\{[5-(4-nitrophenyl)-2-furyl]methylene\}-4,5-dihydroimidazol-4one (10a). Yield 74\%, m.p. 296-298 ${ }^{\circ} \mathrm{C}$. Anal. Calcd. for $\mathrm{C}_{17} \mathrm{H}_{14} \mathrm{~N}_{4} \mathrm{O}_{5}$ (354.3) C, 57.58; H, 3.95; N, 15.81. Found: C, 57.69; H, 3.96; N, 15.66\%. ${ }^{1} \mathrm{H}-\mathrm{NMR}\left(\mathrm{DMSO}-d_{6}\right)\left(\right.$ at $\left.60^{\circ} \mathrm{C}\right), \delta_{\mathrm{H}} 2.14(\mathrm{~s}, 3 \mathrm{H}$, $\left.\mathrm{CH}_{3}\right), 3.67$ (s, 3H, N-CH 3$), 6.58(\mathrm{~s}, 1 \mathrm{H}, \mathrm{H}-6), 7.19\left(\mathrm{~d}, 1 \mathrm{H},{ }^{3} \mathrm{~J}=3.8 \mathrm{~Hz}, \mathrm{H}-4{ }^{\prime}\right), 7.51$ (t, $1 \mathrm{H},{ }^{3} J=3.8$ $\left.\mathrm{Hz},{ }^{4} J=1.8 \mathrm{~Hz}, \mathrm{H}-3^{\prime}\right), 7.78$ (s, $\left.1 \mathrm{H}, \mathrm{NH}\right), 7.97$ (dd, $\left.1 \mathrm{H},{ }^{3} J=9 \mathrm{~Hz},{ }^{4} J=2 \mathrm{~Hz}, \mathrm{H}-11^{\prime}\right), 8.02$ (dd, $\left.1 \mathrm{H},{ }^{3} J=9 \mathrm{~Hz},{ }^{4} J=1.9 \mathrm{~Hz}, \mathrm{H}-7^{\prime}\right), 8.31\left(\mathrm{dd}, 1 \mathrm{H},{ }^{3} \mathrm{~J}=9 \mathrm{~Hz},{ }^{4} J=2.2 \mathrm{~Hz}, \mathrm{H}-10^{\prime}\right), 8.34$ (dd, $1 \mathrm{H},{ }^{3} J=$ $\left.9 \mathrm{~Hz},{ }^{4} \mathrm{~J}=2 \mathrm{~Hz}, \mathrm{H}-8^{\prime}\right)$.

2-Acetylamino-1-methyl-5-\{[5-(2-nitrophenyl)-2-furyl]methylene\}-4,5-dihydroimidazol-4one (10b). Yield 68\%, m.p. 230-231 ${ }^{\circ} \mathrm{C}$. Anal. Calcd. for $\mathrm{C}_{17} \mathrm{H}_{14} \mathrm{~N}_{4} \mathrm{O}_{5}$ (354.3) C, 57.58; H, 3.95; N, 15.81. Found: C, 57.44; H, 3.87; N, 15.69\%. ${ }^{1} \mathrm{H}-\mathrm{NMR}\left(\mathrm{DMSO}-d_{6}\right)$, (heated at $\left.60^{\circ} \mathrm{C}\right), \delta_{\mathrm{H}} 2.12$ (s, 3H, $\left.\mathrm{CH}_{3}\right), 3.25$ (s, 3H, N-CH3), 6.45 (s, 1H, H-6), 7.07 (d, 1H, $\left.{ }^{3} \mathrm{~J}=3.8 \mathrm{~Hz}, \mathrm{H}-3^{\prime}\right), 7.58-7.64$ (m, 1H, H-9') 7.77 (dd, $\left.1 \mathrm{H},{ }^{3} J=8.1 \mathrm{~Hz},{ }^{4} \mathrm{~J}=1.1 \mathrm{~Hz}, \mathrm{H}-7^{\prime}\right), 7.8$ (d, $\left.1 \mathrm{H},{ }^{3} J=3.8 \mathrm{~Hz}, \mathrm{H}-4^{\prime}\right), 7.91-$ 7.95 (m, 2H, H-8', 9'), 11.61 (s, 1H, NH).

2-Acetylamino-1-methyl-5-\{[5-(4-chlorophenyl-2-furyl]methylene\}-4,5-dihydroimidazol-4one (10d). Yield 77\%, m.p. 238-240 ${ }^{\circ} \mathrm{C}$. Anal. Calcd. for $\mathrm{C}_{17} \mathrm{H}_{14} \mathrm{Cl} \mathrm{N}_{3} \mathrm{O}_{3}$ (343.7) C, 59.40; $\mathrm{H}$, 4.10; N, 12.22. Found: C, 59.46; H, 3.96; N, 12.01\%. ${ }^{1} \mathrm{H}-\mathrm{NMR} \delta_{\mathrm{H}}\left(\mathrm{CDCl}_{3}\right) 2.28\left(\mathrm{~s}, 3 \mathrm{H}, \mathrm{CH}_{3}\right)$, $3.79\left(\mathrm{~s}, 2 \mathrm{H}, \mathrm{N}-\mathrm{CH}_{3}\right), 6.43(\mathrm{~s}, 1 \mathrm{H}, \mathrm{H}-6), 6.80\left(\mathrm{~d}, 1 \mathrm{H},{ }^{3} \mathrm{~J}=3.8 \mathrm{~Hz}, \mathrm{H}-4^{\prime}\right), 7.36\left(\mathrm{dd}, 1 \mathrm{H},{ }^{3} \mathrm{~J}=8.8 \mathrm{~Hz}\right.$, $\left.{ }^{4} J=2 \mathrm{~Hz}, \mathrm{H}-8^{\prime}\right), 7.39$ (dd, $\left.1 \mathrm{H},{ }^{3} J=8.8 \mathrm{~Hz},{ }^{4} J=1.9 \mathrm{~Hz}, \mathrm{H}-10 '\right), 7.64\left(\mathrm{dd}, 1 \mathrm{H},{ }^{3} J=8.8 \mathrm{~Hz},{ }^{4} J=2\right.$ Hz, H-7'), 7.67 (dd, $1 \mathrm{H},{ }^{3} J=8.8 \mathrm{~Hz},{ }^{4} J=2 \mathrm{~Hz}, \mathrm{H}-11^{\prime}$ ), 7.97 (d, 1H, ${ }^{3} J=3.8 \mathrm{~Hz}, \mathrm{H}-3^{\prime}$ ), 10.86 (s, $1 \mathrm{H}, \mathrm{NH})$.

5-\{[5-(4-Nitrophenyl)-2-furyl]methylene\}-2-thioxoimidazolidin-4-one (11a). Yield 65\%, m.p. 212-214 ${ }^{\circ} \mathrm{C}$. Anal. Calcd. for $\mathrm{C}_{14} \mathrm{H}_{9} \mathrm{~N}_{3} \mathrm{O}_{4} \mathrm{~S}$ (315.3) C, 53.33; H, 2.88; N, 13.33. Found: C, 53.45; H, 3.16; N, 13.02\%. ${ }^{1} \mathrm{H}-\mathrm{NMR}$ DMSO-d $\left.d_{6}\right) \delta \mathrm{H} 6.45$ (s, 1H, H-6), 7.39 (d, 1H, ${ }^{3} J=3.8 \mathrm{~Hz}, \mathrm{H}-4{ }^{\prime}$ ), $7.53\left(\mathrm{~d}, 1 \mathrm{H},{ }^{3} \mathrm{~J}=3.8 \mathrm{~Hz}, \mathrm{H}-3^{\prime}\right), 8.12\left(\mathrm{dd}, 1 \mathrm{H},{ }^{3} \mathrm{~J}=7 \mathrm{~Hz},{ }^{4} \mathrm{~J}=2 \mathrm{~Hz}, \mathrm{H}-7^{\prime}\right), 8.15\left(\mathrm{dd}, 1 \mathrm{H},{ }^{3} \mathrm{~J}=7 \mathrm{~Hz}\right.$, $\left.{ }^{4} J=2 \mathrm{H}, \mathrm{H}-11^{\prime}\right), 8.34\left(\mathrm{dd}, 1 \mathrm{H},{ }^{3} J=7.1 \mathrm{~Hz},{ }^{4} \mathrm{~J}=2 \mathrm{~Hz}, \mathrm{H}-8^{\prime}\right), 8.37\left(\mathrm{dd}, 1 \mathrm{H},{ }^{3} J=7 \mathrm{~Hz},{ }^{4} \mathrm{~J}=2 \mathrm{~Hz}\right.$, H-10'), 9.71 (s, 1H, NH-3), 12.15 (s, 1H, NH-1).

5-\{[5-(4-Nitrophenyl)-2-furyl]methylene\}-1,3-thiazolidin-2,4-one (12a). Yield 54\%, m.p. 345-346 ${ }^{\circ} \mathrm{C}$. Anal. Calcd. for $\mathrm{C}_{14} \mathrm{H}_{8} \mathrm{~N}_{2} \mathrm{O}_{5} \mathrm{~S}$ (316.3) C, 53.16; H, 2.55; N, 8.86. Found: C, 52.94; H, 2.44; N, 8.64\%. ${ }^{1} \mathrm{H}-\mathrm{NMR}$ (DMSO- $\left.d_{6}\right) \delta_{\mathrm{H}} 7,25$ (d, $\left.1 \mathrm{H},{ }^{3} \mathrm{~J}=3.8 \mathrm{~Hz}, \mathrm{H}-4^{\prime}\right), 7.53\left(\mathrm{~d}, 1 \mathrm{H},{ }^{3} J=3.8\right.$ Hz, H-3') 7.63 (s, 1H, H-6), 7.96 (dd, $\left.1 \mathrm{H},{ }^{3} J=8.3 \mathrm{~Hz},{ }^{4} J=2.1 \mathrm{~Hz}, \mathrm{H}-11^{\prime}\right), 7.99$ (dd, $1 \mathrm{H},{ }^{3} J=$ $\left.8.3, \mathrm{~Hz},{ }^{4} \mathrm{~J}=2.1 \mathrm{~Hz}, \mathrm{H}-7^{\prime}\right), 8.32\left(\mathrm{dd}, 1 \mathrm{H},{ }^{3} \mathrm{~J}=8.9 \mathrm{~Hz},{ }^{4} \mathrm{~J}=1.8 \mathrm{~Hz}, \mathrm{H}-10^{\prime}\right), 8.34\left(\mathrm{dd}, 1 \mathrm{H},{ }^{3} J=8.9\right.$ $\left.\mathrm{Hz},{ }^{4} \mathrm{~J}=1.8 \mathrm{~Hz}, \mathrm{H}-8^{\prime}\right), 12.55$ (s, 1H, NH).

2-\{[5-(4-Nitrophenyl)-2-furyl]methylene\}[1,3]thiazolo[3,2-a]benzimidazol-3(2H)-one (13a). Yield 80\%, m.p. 314-315 ${ }^{\circ}$ C. Anal. Calcd. for $\mathrm{C}_{20} \mathrm{H}_{11} \mathrm{~N}_{3} \mathrm{O}_{4} \mathrm{~S}$ (389.4) C, 61.69; H, 2.85; 10.79 . Found: C, 61.39\%; H, 2.84\%; N, 10.64\%. ${ }^{1} \mathrm{H}-\mathrm{NMR}$ (DMSO- $d_{6}$ ), (heating at $\left.100^{\circ} \mathrm{C}\right), \delta_{\mathrm{H}} 7,38(\mathrm{~d}$, $\left.1 \mathrm{H},{ }^{3} J=3.9 \mathrm{~Hz}, \mathrm{H}-3^{\prime}\right), 7.45$ (d, 1H, ${ }^{3} J=3.9 \mathrm{~Hz}, \mathrm{H}-4^{\prime}$ ), 7.59-7,74 (m, 2H, H-6, 7), 7.89 (dd, 2H, 
$\left.{ }^{3} J=9.2 \mathrm{~Hz},{ }^{4} J=2.2 \mathrm{~Hz}, \mathrm{H}-7^{\prime}, 11^{\prime}\right) .8 .08-8.13(\mathrm{~m}, 2 \mathrm{H}, \mathrm{H}-5,8), 8.16$ (s, 1H, H-10), 8.39 (dd, $2 \mathrm{H},{ }^{3} J$ $\left.=9.2 \mathrm{~Hz},{ }^{4} J=2.2 ; \mathrm{H}-8^{\prime}, 10^{\prime}\right)$.

2-\{[5-(2-Nitrophenyl)-2-furyl]methylene\}[1,3]thiazolo[3,2-a]benzimidazol-3(2H)-one (13b). Yield 74\%, m.p. 281-283 ${ }^{\circ} \mathrm{C}$. Anal. Calcd. for $\mathrm{C}_{20} \mathrm{H}_{11} \mathrm{~N}_{3} \mathrm{O}_{4} \mathrm{~S}(389.4) \mathrm{C}, 61.69 ; \mathrm{H}, 2.85 ; \mathrm{N}, 10.79$; Found: C, 61.54; H, 2.78; N, 10.77\%. ${ }^{1} \mathrm{H}-\mathrm{NMR}\left(\mathrm{DMSO}-d_{6}\right)$, (heating at $\left.60^{\circ} \mathrm{C}\right) \delta_{\mathrm{H}}, 7.29(\mathrm{~d}, 1 \mathrm{H}$, $\left.{ }^{3} J=3.9 \mathrm{~Hz}, \mathrm{H}-3^{\prime}\right), 7.33-7.41\left(\mathrm{~m}, 2 \mathrm{H}, \mathrm{H}-7^{\prime}, 9^{\prime}\right), 7.43$ (d, 1H, $\left.{ }^{3} J=3.9 \mathrm{~Hz}, \mathrm{H}-4^{\prime}\right), 7.65-7,76$ (m, 2H, H-8', 10'), 7.83-7.96 (m, 2H, H-6,7), 7.97 (s, 1H, H-10), 8.01 (d, $\left.1 \mathrm{H},{ }^{3} \mathrm{~J}=8,3 \mathrm{~Hz}, \mathrm{H}-8\right)$, $8.08\left(\mathrm{~d}, 1 \mathrm{H},{ }^{3} J=8,2 \mathrm{~Hz}, \mathrm{H}-5\right)$.

2-\{[5-(4-Bromophenyl)-2-furyl]methylene\}[1,3]thiazolo[3,2-a]benzimidazol-3(2H)-one (13c). Yield 82\%, m.p. $265-266{ }^{\circ} \mathrm{C}$. Anal. Calcd. for $\mathrm{C}_{20} \mathrm{H}_{11} \mathrm{BrN}_{2} \mathrm{O}_{2} \mathrm{~S}$ (423.3) C, 56.75; H, 2.62; $\mathrm{N}, 6.62$. Found: $\left.\mathrm{C}, 56.54 ; \mathrm{H}, 2.46 ; \mathrm{N}, 6.34 \% .{ }^{1} \mathrm{H}-\mathrm{NMR} \mathrm{CDCl}_{3}\right) \delta_{\mathrm{H}}\left(6.89\left(\mathrm{~d}, 1 \mathrm{H},{ }^{3} J=3.7 \mathrm{~Hz}, \mathrm{H}-\right.\right.$ $\left.3^{\prime}\right), 6.97\left(\mathrm{~d}, 1 \mathrm{H},{ }^{3} J=3.7 \mathrm{~Hz}, \mathrm{H}-4^{\prime}\right), 7.27-7,42\left(\mathrm{~m}, 2 \mathrm{H}, \mathrm{H}-8^{\prime} 10^{\prime}\right), 7.59-7.69$ (m, 5H, H$\left.5,6,7,7^{\prime}, 11^{\prime}\right), 7.78$ (s, $\left.1 \mathrm{H}, \mathrm{H}-10\right), 8.03$ (dd, $1 \mathrm{H},{ }^{3} J=7.9 \mathrm{~Hz},{ }^{4} J=1.9 \mathrm{~Hz}, \mathrm{H}-8$ ).

2-\{[5-(4-Chlorophenyl)-2-furyl]methylene\}[1,3]thiazolo[3,2-a]benzimidazol-3(2H)-one (13d). Yield 85\%, m.p. 259-261 ${ }^{\circ} \mathrm{C}$. Anal. Calcd. for $\mathrm{C}_{20} \mathrm{H}_{11} \mathrm{ClN}_{2} \mathrm{O}_{2} \mathrm{~S}$ (378.8) C, 63.41; H, 2.93; N 7.39; Found C, 63.24; H, 2.85; N, 7.15\%. ${ }^{1} \mathrm{H}-\mathrm{NMR}\left(\mathrm{CDCl}_{3}\right) \delta_{\mathrm{H}}, 6.87\left(\mathrm{~d}, 1 \mathrm{H},{ }^{3} J=3.7 \mathrm{~Hz}, \mathrm{H}-\right.$ $\left.3^{\prime}\right), 6.99\left(\mathrm{~d}, 1 \mathrm{H},{ }^{3} J=3.8 \mathrm{~Hz}, \mathrm{H}-4^{\prime}\right), 7.31-7.42\left(\mathrm{~m}, 2 \mathrm{H}, \mathrm{H}-8^{\prime} 10^{\prime}\right), 7.45\left(\mathrm{dd}, 1 \mathrm{H},{ }^{3} J=4.3 \mathrm{~Hz},{ }^{4} J=2\right.$ $\left.\mathrm{Hz}, \mathrm{H}-7^{\prime}\right), 7.48\left(\mathrm{dd}, 1 \mathrm{H},{ }^{3} J=4.4 \mathrm{~Hz},{ }^{4} J=2 \mathrm{~Hz}, \mathrm{H}-11^{\prime}\right), 7.69$ (dd, $1 \mathrm{H},{ }^{3} J=7.4 \mathrm{~Hz},{ }^{4} J=1.6 \mathrm{~Hz}, \mathrm{H}-$ 5), $7.72\left(\mathrm{tt}, 1 \mathrm{H},{ }^{3} J=7.4 \mathrm{~Hz}, 4 \mathrm{~J}=1.8 \mathrm{~Hz},{ }^{4} J=2.3 \mathrm{~Hz}, \mathrm{H}-7\right), 7.76\left(\mathrm{tt}, 1 \mathrm{H},{ }^{3} J=7.4 \mathrm{~Hz},{ }^{4} J=1.6 \mathrm{~Hz}\right.$, $\left.{ }^{4} J=2.3 \mathrm{~Hz}, \mathrm{H}-6\right), 7.81$ (s, $\left.1 \mathrm{H}, \mathrm{H}-10\right), 8.04$ (dd, $\left.1 \mathrm{H},{ }^{3} J=7.4 \mathrm{~Hz},{ }^{4} J=1.8 \mathrm{~Hz}, \mathrm{H}-8\right)$.

4-\{[5-(4-Nitrophenyl)-2-furyl]methylene\}-2-phenyl-1,3-oxazol-5(4H)-one (14a). Yield 68\%, m.p. $238-240{ }^{\circ} \mathrm{C}$. Anal. Calcd. for $\mathrm{C}_{20} \mathrm{H}_{12} \mathrm{~N}_{2} \mathrm{O}_{5}(360.33) \mathrm{C}, 66.67 ; \mathrm{H}, 3.36 ; \mathrm{N}, 7.77$. Found: $\mathrm{C}$, 66.54; H, 3.18\%; N, 7.59\%. ${ }^{1} \mathrm{H}-\mathrm{NMR} \delta_{\mathrm{H}}\left(\mathrm{CDCl}_{3}\right) 7.13\left(\mathrm{~d}, 1 \mathrm{H},{ }^{3} \mathrm{~J}=3.8 \mathrm{~Hz}, \mathrm{H}-3{ }^{\prime}\right) ; 7.19$ (s, $1 \mathrm{H}, \mathrm{H}-$ 6); 7.50-7.68 (m, 3H, H-9, 10, 11); $7.72\left(\mathrm{~d}, 1 \mathrm{H},{ }^{3} J=3.9 \mathrm{~Hz}, \mathrm{H}-4{ }^{\prime}\right) ; 7.91\left(\mathrm{~d}, 1 \mathrm{H},{ }^{3} J=8.8 \mathrm{~Hz}, \mathrm{H}-\right.$ $\left.8^{\prime}\right) ; 7.94\left(\mathrm{~d}, 1 \mathrm{H},{ }^{3} J=8.8 \mathrm{~Hz}, \mathrm{H}-10^{\prime}\right) ; 8.17$ (dd, $\left.1 \mathrm{H},{ }^{3} J=7.1 \mathrm{~Hz},{ }^{4} J=1.9 \mathrm{~Hz}, \mathrm{H}-12\right) ; 8.19$ (dd, $1 \mathrm{H}$, $\left.{ }^{3} J=7.1 \mathrm{~Hz},{ }^{4} J=1.9 \mathrm{~Hz}, \mathrm{H}-8\right) ; 8.30\left(\mathrm{~d}, 1 \mathrm{H},{ }^{3} J=8.8 \mathrm{~Hz}, \mathrm{H}-7^{\prime}\right) ; 8.33$ (d, $\left.1 \mathrm{H},{ }^{3} J=8.8 \mathrm{~Hz}, \mathrm{H}-11^{\prime}\right)$.

4-\{[5-(4-Bromophenyl)-2-furyl]methylene\}-2-phenyl-1,3-oxazol-5(4H)-one (14b). Yield 70\%, m.p. 209-211 ${ }^{\circ} \mathrm{C}$. Anal. Calcd. for $\mathrm{C}_{20} \mathrm{H}_{12} \mathrm{BrNO}_{3}$ (394.2) C, 60.94; H, 3.07; Br, 20.27; N, 3.55. Found: C, 61.05; H, 3.11; Br, 20.24; N, 3.32\%. ${ }^{1} \mathrm{H}-\mathrm{NMR}\left(\mathrm{CDCl}_{3}\right) \delta_{\mathrm{H}} 6.93\left(\mathrm{~d}, 1 \mathrm{H},{ }^{3} J=3.8 \mathrm{~Hz}, \mathrm{H}-\right.$ $\left.3^{\prime}\right), 7.21$ (s, $\left.1 \mathrm{H}, \mathrm{H}-6\right), 7.51\left(\mathrm{dd}, 1 \mathrm{H},{ }^{3} J=6.8 \mathrm{~Hz},{ }^{4} J=2 \mathrm{~Hz}, \mathrm{H}-8^{\prime}\right), 7.53\left(\mathrm{dd}, 1 \mathrm{H},{ }^{3} J=6.8 \mathrm{~Hz},{ }^{4} J=\right.$ $\left.2 \mathrm{~Hz}, \mathrm{H}-10^{\prime}\right), 7.56\left(\mathrm{~d}, 1 \mathrm{H},{ }^{3} J=3.8 \mathrm{~Hz}, \mathrm{H}-4^{\prime}\right), 7.59\left(\mathrm{~d}, 1 \mathrm{H},{ }^{3} J=7 \mathrm{~Hz}, \mathrm{H}-11^{\prime}\right), 7.63\left(\mathrm{~d}, 1 \mathrm{H},{ }^{3} J=7\right.$ $\left.\mathrm{Hz}, \mathrm{H}-7^{\prime}\right), 7.66-7.71$ (m, 3H, H-9, 10, 11), 8.16 (dd, $\left.1 \mathrm{H},{ }^{3} J=7.1 \mathrm{~Hz},{ }^{4} J=1.9 \mathrm{~Hz}, \mathrm{H}-12\right), 8.18$ (dd, $\left.1 \mathrm{H},{ }^{3} J=7.1 \mathrm{~Hz},{ }^{4} J=1.9 \mathrm{~Hz}, \mathrm{H}-8\right)$.

4-\{[5-(4-Chlorophenyl)-2-furyl]methylene\}-2-phenyl-1,3-oxazol-5(4H)-one (14c). Yield 70\%, m.p. $210-212{ }^{\circ} \mathrm{C}$. Anal. Calcd. for $\mathrm{C}_{20} \mathrm{H}_{12} \mathrm{ClNO}_{3}$ (349.7) C, 68.68; H, 3.46; Cl, 10.14; N, 4.00 . Found: C, 68.55; H, 3.28; Cl, 9.87; N, 4.28\%. ${ }^{1} \mathrm{H}-\mathrm{NMR}\left(\mathrm{CDCl}_{3}\right) \delta_{\mathrm{H}} 6.92\left(\mathrm{~d}, 1 \mathrm{H},{ }^{3} J=3.8 \mathrm{~Hz}, \mathrm{H}-\right.$ $\left.3^{\prime}\right), 7.20$ (s, $\left.1 \mathrm{H}, \mathrm{H}-6\right), 7.41$ (dd, $\left.1 \mathrm{H},{ }^{3} J=6.8 \mathrm{~Hz},{ }^{4} J=2 \mathrm{~Hz}, \mathrm{H}-8^{\prime}\right), 7.44\left(\mathrm{dd}, 1 \mathrm{H},{ }^{3} J=6.8 \mathrm{~Hz},{ }^{4} J=\right.$ $\left.2 \mathrm{~Hz}, \mathrm{H}-10^{\prime}\right), 7.5-7.65$ (m, 3H, H-9, 10, 11), $7.68\left(\mathrm{~d}, 1 \mathrm{H},{ }^{3} J=3.9 \mathrm{~Hz}, \mathrm{H}-4^{\prime}\right), 7.71\left(\mathrm{~d}, 1 \mathrm{H},{ }^{3} J=\right.$ $\left.7 \mathrm{~Hz}, \mathrm{H}-11^{\prime}\right), 7.74\left(\mathrm{~d}, 1 \mathrm{H},{ }^{3} J=7 \mathrm{~Hz}, \mathrm{H}-7^{\prime}\right), 8.16\left(\mathrm{dd}, 1 \mathrm{H},{ }^{3} J=7.1 \mathrm{~Hz},{ }^{4} J=1.9 \mathrm{~Hz}, \mathrm{H}-12\right), 8.18$ (dd, $1 \mathrm{H},{ }^{3} J=7.1 \mathrm{~Hz},{ }^{4} J=1.9 \mathrm{~Hz}, \mathrm{H}-8$ ). 
4-\{[5-(4-Nitrophenyl)-2-furyl]methylene\}-2-(3-nitrophenyl)-1,3-oxazol-5(4H)-one (14d). Yield 54\%, m.p. 259-261 ${ }^{\circ} \mathrm{C}$. Anal. Calcd. for $\mathrm{C}_{20} \mathrm{H}_{11} \mathrm{~N}_{3} \mathrm{O}_{7}$ (405.3) C, 59.27; H, 2.74; N, 10.37. Found: C, 59.04; H, 2.59; N, 10.19\%. ${ }^{1} \mathrm{H}-\mathrm{NMR}\left(\mathrm{CDCl}_{3}\right) \delta_{\mathrm{H}} 7.32(\mathrm{~s}, 1 \mathrm{H}, \mathrm{H}-6), 7.73\left(\mathrm{~d}, 1 \mathrm{H},{ }^{3} J=3.8 \mathrm{~Hz}\right.$, H-4'), 7.77(d, 1H, $\left.{ }^{3} J=3.8 \mathrm{~Hz}, \mathrm{H}-3^{\prime}\right), 7.77$ (t, $\left.1 \mathrm{H},{ }^{3} J=7.1 \mathrm{~Hz}, \mathrm{H}-11\right), 7.95$ (dd, $1 \mathrm{H},{ }^{3} J=9 \mathrm{~Hz}, \mathrm{H}-$ $\left.8^{\prime}\right), 7.98\left(\mathrm{~d}, 1 \mathrm{H},{ }^{3} J=9 \mathrm{~Hz}, \mathrm{H}-10^{\prime}\right), 8.33\left(\mathrm{~d}, 1 \mathrm{H},{ }^{3} J=9 \mathrm{~Hz}, \mathrm{H}-7^{\prime}\right), 8.36$ (d, $\left.1 \mathrm{H},{ }^{3} J=9 \mathrm{~Hz}, \mathrm{H}-11^{\prime}\right)$, $8.45\left(\mathrm{dd}, 2 \mathrm{H},{ }^{3} \mathrm{~J}=8.5 \mathrm{~Hz},{ }^{4} \mathrm{~J}=1.9 \mathrm{~Hz}, \mathrm{H}-10,12\right), 9.05$ (s, 1H, H-8).

4-\{[5-(4-Bromophenyl)-2-furyl]methylene\}-2-(3-nitrophenyl)-1,3-oxazol-5(4H)-one

(14e). Yield 68\%, m.p. $230-232{ }^{\circ} \mathrm{C}$. Anal. Calcd. for $\mathrm{C}_{20} \mathrm{H}_{11} \mathrm{BrN}_{2} \mathrm{O}_{5}(439.2) \mathrm{C}, 54.69 ; \mathrm{H}, 2.52 ; \mathrm{N}, 6.38$; $\mathrm{Br}, 18.19$. Found: C, 54.68; H, 2.51; N, 6.38; Br, 17.83\%. ${ }^{1} \mathrm{H}-\mathrm{NMR}\left(\mathrm{CDCl}_{3}\right) \delta_{\mathrm{H}} 6.98\left(\mathrm{~d}, 1 \mathrm{H},{ }^{3} J\right.$ $\left.=3.8 \mathrm{~Hz}, \mathrm{H}-3^{\prime}\right), 7.28(\mathrm{~s}, 1 \mathrm{H}, \mathrm{H}-6), 7.59$ (dd, $\left.1 \mathrm{H},{ }^{3} \mathrm{~J}=8.4 \mathrm{~Hz},{ }^{4} \mathrm{~J}=1.9 \mathrm{~Hz}, \mathrm{H}-8^{\prime}\right), 7.62\left(\mathrm{dd}, 1 \mathrm{H},{ }^{3} J=\right.$ $\left.8.4 \mathrm{~Hz},{ }^{4} \mathrm{~J}=1.9 \mathrm{~Hz}, \mathrm{H}-10^{\prime}\right), 7.75\left(\mathrm{t}, 1 \mathrm{H},{ }^{3} \mathrm{~J}=7.5 \mathrm{~Hz} \mathrm{H}-11\right), 7.71$ (d, $\left.1 \mathrm{H},{ }^{3} J=3.9 \mathrm{~Hz}, \mathrm{H}-4^{\prime}\right), 7.67$ $\left(\mathrm{dd}, 1 \mathrm{H},{ }^{3} \mathrm{~J}=8.6 \mathrm{~Hz},{ }^{4} \mathrm{~J}=2 \mathrm{~Hz}, \mathrm{H}-7^{\prime}\right), 7.70\left(\mathrm{dd}, 1 \mathrm{H},{ }^{3} \mathrm{~J}=8.6 \mathrm{~Hz},{ }^{4} \mathrm{~J}=1.9 \mathrm{~Hz}, \mathrm{H}-11^{\prime}\right), 8.43$ (t, $2 \mathrm{H}$, $\left.{ }^{3} J=7.5 \mathrm{~Hz}, \mathrm{H}-10,12\right), 9.03$ (s, 1H, H-8).

4-\{[5-(4-Chlorophenyl)-2-furyl]methylene\}-2-(3-nitrophenyl)-1,3-oxazol-5(4H)-one (14f). Yield 72\%, m.p. $217-219^{\circ} \mathrm{C}$. Anal. Calcd. for $\mathrm{C}_{20} \mathrm{H}_{11} \mathrm{ClN}_{2} \mathrm{O}_{5}(394.7) \mathrm{C}, 60.85 ; \mathrm{H}, 2.81 ; \mathrm{Cl}, 8.98$; $\mathrm{N}, 7.10$. Found: C, 60.64; H, 2.98; Cl, 9.14; N, 6.83\%. ${ }^{1} \mathrm{H}-\mathrm{NMR}\left(\mathrm{CDCl}_{3}\right) \delta_{\mathrm{H}} 6.96\left(\mathrm{~d}, 1 \mathrm{H},{ }^{3} J=3.8\right.$ Hz, H-3') 7.28 (s, 1H, H-6), 7.46 (dd, $\left.1 \mathrm{H},{ }^{3} J=8.4 \mathrm{~Hz},{ }^{4} J=1.9 \mathrm{~Hz}, \mathrm{H}-8^{\prime}\right), 7.43$ (dd, $1 \mathrm{H},{ }^{3} J=8.4$ $\left.\mathrm{Hz},{ }^{4} J=1.9 \mathrm{~Hz}, \mathrm{H}-10^{\prime}\right), 7.5-7.6(\mathrm{~m}, 1 \mathrm{H}, \mathrm{H}-11), 7.70$ (d, $\left.1 \mathrm{H},{ }^{3} J=3.9 \mathrm{~Hz}, \mathrm{H}-4^{\prime}\right), 7.74\left(\mathrm{dd}, 1 \mathrm{H},{ }^{3} J\right.$ $\left.=8.8 \mathrm{~Hz},{ }^{4} \mathrm{~J}=1.9 \mathrm{~Hz}, \mathrm{H}-7^{\prime}\right), 7.77\left(\mathrm{~d}, 1 \mathrm{H},{ }^{3} \mathrm{~J}=8.8 \mathrm{~Hz},{ }^{4} \mathrm{~J}=1.9 \mathrm{~Hz}, \mathrm{H}-11^{\prime}\right), 8.43\left(\mathrm{t}, 2 \mathrm{H},{ }^{3} J=7.5\right.$ $\mathrm{Hz}, \mathrm{H}-10,12), 9.02$ (s, 1H, H-8).

4-\{[5-(4-Chlorophenyl)-2-furyl]methylene\}-2-(4-nitrophenyl)-1,3-oxazol-5(4H)-one

(14g). Yield 78\% m.p. $310-312{ }^{\circ} \mathrm{C}$. $220-222^{\circ} \mathrm{C}$. Anal. Calcd. for $\mathrm{C}_{20} \mathrm{H}_{11} \mathrm{ClN}_{2} \mathrm{O}_{5}$ (394.7) C, 60.85; H, 2.81; N 7.10. Found: C, 60.54; H, 2.98; N, 7.09\%. ${ }^{1} \mathrm{H}-\mathrm{NMR}$ (DMSO- $\left.d_{6}\right) \delta_{\mathrm{H}} 7.35$ (s, 1H, H-6), $7.45\left(\mathrm{~d}, 1 \mathrm{H},{ }^{3} \mathrm{~J}=3.7 \mathrm{~Hz}, \mathrm{H}-4^{\prime}\right), 7.60\left(\mathrm{dd}, 1 \mathrm{H},{ }^{3} \mathrm{~J}=8.6 \mathrm{~Hz},{ }^{4} J=2.2 \mathrm{~Hz}, \mathrm{H}-8^{\prime}\right), 7.63\left(\mathrm{dd}, 1 \mathrm{H},{ }^{3} J=\right.$ $\left.8.6 \mathrm{~Hz},{ }^{4} \mathrm{~J}=2.2 \mathrm{~Hz}, \mathrm{H}-10^{\prime}\right), 7.74\left(\mathrm{~d}, 1 \mathrm{H},{ }^{3} \mathrm{~J}=3.8 \mathrm{~Hz}, \mathrm{H}-3^{\prime}\right), 7.92\left(\mathrm{dd}, 1 \mathrm{H},{ }^{3} \mathrm{~J}=8.6 \mathrm{~Hz},{ }^{4} \mathrm{~J}=2.2\right.$ $\left.\mathrm{Hz}, \mathrm{H}^{-} 7^{\prime}\right), 7.95$ (dd, $\left.1 \mathrm{H},{ }^{3} \mathrm{~J}=8.6 \mathrm{~Hz},{ }^{4} \mathrm{~J}=2.2 \mathrm{~Hz}, \mathrm{H}-11^{\prime}\right), 8.33\left(\mathrm{~d}, 1 \mathrm{H},{ }^{3} \mathrm{~J}=8,2 \mathrm{~Hz}, \mathrm{H}-8\right), 8.36$ (d, $\left.1 \mathrm{H},{ }^{3} J=8.2 \mathrm{~Hz}, \mathrm{H}-12\right), 8.42\left(\mathrm{~d}, 1 \mathrm{H},{ }^{3} \mathrm{~J}=8.2 \mathrm{~Hz}, \mathrm{H}-9\right), 8.45$ (d, $\left.1 \mathrm{H},{ }^{3} J=8.2 \mathrm{~Hz}, \mathrm{H}-11\right)$.

2-(4'-Acetylaminophenyl)-4-\{[5-(4-chlorophenyl)-2-furyl]methylene\}-1,3-oxazol-5(4H)-one (14h). Yield 61\%, m.p. 310-312 ${ }^{\circ} \mathrm{C}$. Anal. Calcd. for $\mathrm{C}_{22} \mathrm{H}_{15} \mathrm{ClN}_{2} \mathrm{O}_{4}$ (406.8) C, 64.95; H, 3.72; $\mathrm{Cl}, 8.71 ; \mathrm{N}, 6.89$. Found: C, 64.74; H, 3.68; Cl, 8.41; N, 6.59\%. ${ }^{1} \mathrm{H}-\mathrm{NMR}\left(\mathrm{DMSO}-d_{6}\right) \delta_{\mathrm{H}} 7.14(\mathrm{~s}$, $1 \mathrm{H}, \mathrm{H}-6), 7.32$ (d, $\left.1 \mathrm{H},{ }^{3} J=3.7 \mathrm{~Hz}, \mathrm{H}-3^{\prime}\right), 7.55$ (d, $\left.1 \mathrm{H},{ }^{3} J=8.4 \mathrm{~Hz}, \mathrm{H}-8^{\prime}\right), 7.58$ (d, $1 \mathrm{H},{ }^{3} J=8.4$ $\left.\mathrm{Hz}, \mathrm{H}-10^{\prime}\right), 7.64$ (d, $\left.1 \mathrm{H},{ }^{3} \mathrm{~J}=3.9 \mathrm{~Hz}, \mathrm{H}-4^{\prime}\right), 7.81$ (d, $\left.1 \mathrm{H},{ }^{3} \mathrm{~J}=8.4 \mathrm{~Hz}, \mathrm{H}-7^{\prime}\right), 7.84$ (d, $1 \mathrm{H},{ }^{3} J=8.4$ $\mathrm{Hz}, \mathrm{H}-11^{\prime}$ ), 7.87 (d, $\left.1 \mathrm{H},{ }^{3} J=8.8 \mathrm{~Hz}, \mathrm{H}-9\right), 7.90$ (d, $\left.1 \mathrm{H},{ }^{3} \mathrm{~J}=8 \mathrm{~Hz}, \mathrm{H}-10\right), 8.05$ (d, $1 \mathrm{H},{ }^{3} J=8.1$ $\mathrm{Hz}, \mathrm{H}-12), 8.08$ (d, $\left.1 \mathrm{H},{ }^{3} \mathrm{~J}=8.8 \mathrm{~Hz}, \mathrm{H}-8\right)$.

5-(2,5-Dimethoxyphenyl)-3-\{[5-(4-nitrophenyl)-2-furyl]methylene\}furan-2(3H)-one (15a). Yield 65\%, m.p. 314-315 ${ }^{\circ} \mathrm{C}$. Anal. Calcd. for $\mathrm{C}_{23} \mathrm{H}_{17} \mathrm{NO}_{7}(419.4) \mathrm{C}, 65.87 \%$; H, 4.09\%; N, 3.34\%; Found: C, 65.86\% H, 4.00\%; N, 3.39\%. ${ }^{1} \mathrm{H}-\mathrm{NMR}\left(\mathrm{DMSO}-d_{6}\right)$, (heating at $\left.100^{\circ} \mathrm{C}\right) \delta_{\mathrm{H}}$ $3.79\left(\mathrm{~s}, 3 \mathrm{H}, 10-\mathrm{OCH}_{3}\right), 4.01\left(\mathrm{~s}, 3 \mathrm{H}, 7-\mathrm{OCH}_{3}\right), 7.6\left(\mathrm{~d}, 1 \mathrm{H},{ }^{3} \mathrm{~J}=3.9 \mathrm{~Hz}, \mathrm{H}-4\right), 7.74\left(\mathrm{~d}, 1 \mathrm{H},{ }^{3} J=\right.$ $\left.3.9 \mathrm{~Hz}, \mathrm{H}-3^{\prime}\right)$ ) $7.72-7.73$ (m, 4H, H-4, 8, 9, 11), 8.13-8.15 (m, 1H, H-6), 8.35 (dd, $2 \mathrm{H},{ }^{3} J=6.8$ $\left.\mathrm{Hz},{ }^{4} \mathrm{~J}=2 \mathrm{~Hz}, \mathrm{H}-8^{\prime}, 10^{\prime}\right), 8.38\left(\mathrm{dd}, 2 \mathrm{H},{ }^{3} \mathrm{~J}=6.5 \mathrm{~Hz},{ }^{4} \mathrm{~J}=2 \mathrm{~Hz}, \mathrm{H}-7^{\prime}, 11^{\prime}\right)$. 
5-(2,5-Dimethoxyphenyl)-3-\{[5-(2-nitrophenyl)-2-furyl]methylene\}furan-2(3H)-one (15b). Yield 60\%, m.p. 199-200 ${ }^{\circ} \mathrm{C}$. Anal. Calcd. for $\mathrm{C}_{23} \mathrm{H}_{17} \mathrm{NO}_{7}$ (419.4) C, 65.87; H, 4.09; N, 3.34; Found: C, 64.98; H, 4.25; N, 3.12\%. $\left.{ }^{1} \mathrm{H}-\mathrm{NMR} \mathrm{CDCl}_{3}\right) \delta_{\mathrm{H}} 3.82\left(\mathrm{~s}, 3 \mathrm{H}, 10-\mathrm{OCH}_{3}\right), 3.97(\mathrm{~s}, 3 \mathrm{H}, 7-$ $\left.\mathrm{OCH}_{3}\right), 6.85\left(\mathrm{~d}, 1 \mathrm{H},{ }^{3} \mathrm{~J}=3.9 \mathrm{~Hz}, \mathrm{H}-4^{\prime}\right), 6.86\left(\mathrm{~d}, 1 \mathrm{H},{ }^{3} \mathrm{~J}=3.9 \mathrm{~Hz}, \mathrm{H}-3^{\prime}\right), 6.93-6.94$ (m, 3H, H-8, 9,11), 7.06 (s, 1H, H-4); 7.37-7.38 (m, 1H, H- 6), 7.53 (tt, $1 \mathrm{H},{ }^{3} J=8.1 \mathrm{~Hz},{ }^{4} J=1.2 \mathrm{~Hz}, \mathrm{H}-9^{\prime}$ ), $7.67\left(\mathrm{tt}, 1 \mathrm{H},{ }^{3} \mathrm{~J}=6.5 \mathrm{~Hz},{ }^{4} \mathrm{~J}=2 \mathrm{~Hz}, \mathrm{H}-10^{\prime}\right), 7.78\left(\mathrm{dd}, 1 \mathrm{H},{ }^{3} \mathrm{~J}=7.5 \mathrm{~Hz},{ }^{4} \mathrm{~J}=1.2 \mathrm{~Hz}, \mathrm{H}-8^{\prime}\right), 7.87$ (dd, $1 \mathrm{H},{ }^{3} \mathrm{~J}=6.5 \mathrm{~Hz},{ }^{4} \mathrm{~J}=1.2 \mathrm{~Hz}, \mathrm{H}-11^{\prime}$ ).

3-\{[5-(4-Bromophenyl)-2-furyl]methylene\}-5-(2,5-dimethoxyphenyl)furan-2(3H)-one (15c). Yield 64\%, m.p. 199-200 ${ }^{\circ} \mathrm{C}$. Anal. Calcd. for $\mathrm{C}_{23} \mathrm{H}_{17} \mathrm{BrO}_{5}(453.3) \mathrm{C}, 60.94 ; \mathrm{H}, 3.78 ; \mathrm{Br}, 17.63$. Found: C, 61.05; H, 3.70; Br, 17.67\%. ${ }^{1} \mathrm{H}-\mathrm{NMR}\left(\mathrm{CDCl}_{3}\right) \delta_{\mathrm{H}} 3.78\left(\mathrm{~s}, 3 \mathrm{H}, 10-\mathrm{OCH}_{3}\right), 4.02(\mathrm{~s}, 3 \mathrm{H}$, $\left.7-\mathrm{OCH}_{3}\right), 6.85\left(\mathrm{~d}, 1 \mathrm{H},{ }^{3} \mathrm{~J}=3.9 \mathrm{~Hz}, \mathrm{H}-4^{\prime}\right), 6.87\left(\mathrm{~d}, 1 \mathrm{H},{ }^{3} \mathrm{~J}=3.9 \mathrm{~Hz}, \mathrm{H}-3{ }^{\prime}\right), 6.95-6.96(\mathrm{~m}, 3 \mathrm{H}, \mathrm{H}-$ 8, 9,11), 7.07 (s, 1H, H-4), 7.38 (t, $\left.1 \mathrm{H},{ }^{4} \mathrm{~J}=1.8 \mathrm{~Hz}, \mathrm{H}-6\right), 7.57$ (dd, $1 \mathrm{H},{ }^{3} \mathrm{~J}=6.8 \mathrm{~Hz},{ }^{4} \mathrm{~J}=2 \mathrm{~Hz}, \mathrm{H}-$ $\left.8^{\prime}\right), 7.60\left(\mathrm{dd}, 1 \mathrm{H},{ }^{3} J=6.5 \mathrm{~Hz},{ }^{4} J=2 \mathrm{~Hz}, \mathrm{H}-10^{\prime}\right), 7.66\left(\mathrm{dd}, 1 \mathrm{H},{ }^{3} J=6.6 \mathrm{~Hz},{ }^{4} J=2 \mathrm{~Hz}, \mathrm{H}-7^{\prime}\right), 7.69$ (dd, $1 \mathrm{H},{ }^{3} \mathrm{~J}=6.5 \mathrm{~Hz},{ }^{4} \mathrm{~J}=2 \mathrm{~Hz}, \mathrm{H}-11^{\prime}$ ).

3-\{[5-(4-Chlorophenyl)-2-furyl]methylene\}-5-(2,5-dimethoxyphenyl)furan-2(3H)-one (15d). Yield 62\%, m.p. 192-193 ${ }^{\circ} \mathrm{C}$. Anal. Calcd. for $\mathrm{C} 23 \mathrm{H} 17 \mathrm{ClO} 5$ (408.8) C, 67.57; H, 4.19; $\mathrm{Cl}$, 8.67. Found: C, 67.32; H, 4.08; Cl, 8.67\%. ${ }^{1} \mathrm{H}-\mathrm{NMR}\left(\mathrm{CDCl}_{3}\right) \delta_{\mathrm{H}} 3.83\left(\mathrm{~s}, 3 \mathrm{H}, 10-\mathrm{OCH}_{3}\right), 4.03$ $\left(\mathrm{s}, 3 \mathrm{H}, 7-\mathrm{OCH}_{3}\right), 6.84\left(\mathrm{~d}, 1 \mathrm{H},{ }^{3} \mathrm{~J}=3.9 \mathrm{~Hz}, \mathrm{H}-4{ }^{\prime}\right), 6.87\left(\mathrm{~d}, 1 \mathrm{H},{ }^{3} \mathrm{~J}=3.9 \mathrm{~Hz}, \mathrm{H}-3\right.$ '), $6.94-6.96$ (m, $3 \mathrm{H}, \mathrm{H}-8,9,11), 7.06$ (s, 1H, H-4), 7.37 (t, 1H, $\left.{ }^{4} \mathrm{~J}=1.8 \mathrm{~Hz}, \mathrm{H}-6\right), 7.41$ (dd, $1 \mathrm{H},{ }^{3} J=6.8 \mathrm{~Hz},{ }^{4} J=2$ $\left.\mathrm{Hz}, \mathrm{H}-8^{\prime}\right), 7.44\left(\mathrm{dd}, 1 \mathrm{H},{ }^{3} J=6.5 \mathrm{~Hz},{ }^{4} J=2 \mathrm{~Hz}, \mathrm{H}-10^{\prime}\right), 7.72\left(\mathrm{dd}, 1 \mathrm{H},{ }^{3} J=6.6 \mathrm{~Hz},{ }^{4} J=2 \mathrm{~Hz}, \mathrm{H}-\right.$ $\left.7^{\prime}\right), 7.75\left(\mathrm{dd}, 1 \mathrm{H},{ }^{3} J=6.5 \mathrm{~Hz},{ }^{4} J=2 \mathrm{~Hz}, \mathrm{H}-11^{\prime}\right)$.

3-\{[5-(4-Chlorophenyl)-2-furyl]methylene\}-5-(2-methoxy-5-methylphenyl)furan-2(3H)-one (15e). Yield 71\%, m.p. $198-199{ }^{\circ} \mathrm{C}$. Anal. Calcd. for $\mathrm{C}_{23} \mathrm{H}_{17} \mathrm{ClO}_{4}(392.8) \mathrm{C}, 70.32 ; \mathrm{H}, 4.36$; $\mathrm{Cl}$, 9.02. Found: $\mathrm{C}, 70.22 ; \mathrm{H}, 4.28 ; \mathrm{Cl}, 8.97 \% .{ }^{1} \mathrm{H}-\mathrm{NMR}\left(\mathrm{CDCl}_{3}\right) \delta_{\mathrm{H}} 2.33\left(\mathrm{~s} 3 \mathrm{H}, 10-\mathrm{CH}_{3}\right), 4.05(\mathrm{~s}$, $\left.3 \mathrm{H}, 7-\mathrm{OCH}_{3}\right), 6.84\left(\mathrm{~d}, 1 \mathrm{H},{ }^{3} \mathrm{~J}=3.8 \mathrm{~Hz}, \mathrm{H}-4^{\prime}\right), 6.85\left(\mathrm{~d}, 1 \mathrm{H},{ }^{3} \mathrm{~J}=3.9 \mathrm{~Hz}, \mathrm{H}-3\right.$ ) $), 6.90\left(\mathrm{~d}, 1 \mathrm{H},{ }^{3} J=\right.$ $8.6 \mathrm{~Hz} \mathrm{H}-8), 7.05$ (s, 1H, H-4), 7.19 (dd, $\left.1 \mathrm{H},{ }^{3} J=8.4 \mathrm{~Hz},{ }^{4} J=2 \mathrm{~Hz} \mathrm{H}-9\right), 7.41$ (dd, $1 \mathrm{H},{ }^{3} J=6.5$ $\left.\mathrm{Hz},{ }^{4} J=2 \mathrm{~Hz}, \mathrm{H}-8^{\prime}\right), 7.44\left(\mathrm{dd}, 1 \mathrm{H},{ }^{3} J=6.5 \mathrm{~Hz},{ }^{4} J=2 \mathrm{~Hz}, \mathrm{H}-10^{\prime}\right), 7.58$ (s, 1H, H-11), 7.67 (d, $\left.1 \mathrm{H},{ }^{4} \mathrm{~J}=2 \mathrm{~Hz}, \mathrm{H}-6\right), 7.72\left(\mathrm{dd}, 1 \mathrm{H},{ }^{3} \mathrm{~J}=7 \mathrm{~Hz},{ }^{4} \mathrm{~J}=2 \mathrm{~Hz}, \mathrm{H}-7^{\prime}\right), 7.75\left(\mathrm{dd}, 1 \mathrm{H},{ }^{3} \mathrm{~J}=6.9 \mathrm{~Hz},{ }^{4} \mathrm{~J}=2\right.$ $\left.\mathrm{Hz}, \mathrm{H}-11^{\prime}\right)$.

\section{Synthesis of compounds 16}

Compound $15(4 \mathrm{mmol})$ was stirred in warm $\left(100-110^{\circ} \mathrm{C}\right)$ formamide $(6 \mathrm{~mL})$ for one hour. Then the mixture was cooled, diluted with water $(50 \mathrm{~mL})$, and the product removed by filtration and crystallized from ethanol.

5-(2,5-Dimethoxyphenyl)-3-\{[5-(4-nitrophenyl)-2-furyl]methylene\}pyrrol-2(3H)-one (16a). Yield 54\% m.p. 323-325 ${ }^{\circ} \mathrm{C}$ (ethanol). Anal. Calcd. for $\mathrm{C}_{23} \mathrm{H}_{18} \mathrm{~N}_{2} \mathrm{O}_{6}(418.4): \mathrm{C}, 66.02 ; \mathrm{H}, 4.34 ; \mathrm{N}$, 6.70. Found: $\mathrm{C}, 66.11 ; \mathrm{H}, 4.34 ; \mathrm{N}, 6.78 \% .{ }^{1} \mathrm{H}-\mathrm{NMR}\left(\mathrm{DMSO}-d_{6}\right)$ (heating at $\left.100^{\circ} \mathrm{C}\right), \delta_{\mathrm{H}} 3.79(\mathrm{~s}$, $\left.3 \mathrm{H}, 10-\mathrm{OCH}_{3}\right), 4.01\left(\mathrm{~s}, 3 \mathrm{H}, 7-\mathrm{OCH}_{3}\right), 6.97$ (s, 1H, H-4), 7.14-7.18 (m, 4H, H-6, 8, 9, 11), 7.23 $\left(\mathrm{d}, 1 \mathrm{H},{ }^{3} J=3.9 \mathrm{~Hz}, \mathrm{H}-3^{\prime}\right), 7.29$ (d, $\left.1 \mathrm{H},{ }^{3} J=3.9 \mathrm{~Hz}, \mathrm{H}-4^{\prime}\right), 7.58$ (brs, $\left.1 \mathrm{H}, \mathrm{NH}\right), 8.07$ (dd, $1 \mathrm{H},{ }^{3} J$ 
$\left.=7 \mathrm{~Hz},{ }^{4} J=2 \mathrm{~Hz}, \mathrm{H}-8^{\prime}\right), 8.08\left(\mathrm{dd}, 1 \mathrm{H},{ }^{3} J=6.9 \mathrm{~Hz},{ }^{4} J=2 \mathrm{~Hz}, \mathrm{H}-10^{\prime}\right), 8.36\left(\mathrm{dd}, 1 \mathrm{H},{ }^{3} J=7 \mathrm{~Hz},{ }^{4} J\right.$ $\left.=2 \mathrm{~Hz}, \mathrm{H}-7^{\prime}\right), 8.39$ (dd, $\left.1 \mathrm{H},{ }^{3} J=6.9 \mathrm{~Hz},{ }^{4} J=2 \mathrm{~Hz}, \mathrm{H}-11^{\prime}\right)$.

\section{3-\{[5-(4-Chlorophenyl)-2-furyl]methylene\}-5-(2,5-dimethoxyphenyl)pyrrol-2(3H)-one}

(16d). Yield 58\%, m.p. $192-193{ }^{\circ} \mathrm{C}$ (ethanol). Anal. Calcd. for $\mathrm{C}_{23} \mathrm{H}_{18} \mathrm{ClNO}_{4}(407.8) \mathrm{C}, 67.73$; $\mathrm{H}, 4.45 ; \mathrm{Cl}, 8.69 ; \mathrm{N}, 3.43$. Found: C, 67.52; $\mathrm{H}, 4.28 ; \mathrm{Cl}, 8.47 ; \mathrm{N} 3.38 \% .{ }^{1} \mathrm{H}-\mathrm{NMR}\left(\mathrm{CDCl}_{3}\right) \delta_{\mathrm{H}}$ $3.82\left(\mathrm{~s}, 3 \mathrm{H}, 10-\mathrm{OCH}_{3}\right), 4.02\left(\mathrm{~s}, 3 \mathrm{H}, 7-\mathrm{OCH}_{3}\right), 6.83\left(\mathrm{~d}, 1 \mathrm{H},{ }^{3} J=3.8 \mathrm{~Hz}, \mathrm{H}-3^{\prime}\right), 6.84\left(\mathrm{~d}, 1 \mathrm{H},{ }^{3} J=\right.$ $3.8 \mathrm{~Hz}, \mathrm{H}-4^{\prime}$ ), 6.93 (s, 2H, H-8, 9), 7.03 (s, 1H, H-11), 7.35 (s, 1H, H-4), 7.39 (dd, $1 \mathrm{H},{ }^{3} J=6.8$ $\left.\mathrm{Hz},{ }^{4} J=2 \mathrm{~Hz}, \mathrm{H}-8^{\prime}\right), 7.43$ (dd, $1 \mathrm{H},{ }^{3} J=6.8 \mathrm{~Hz},{ }^{4} J=2 \mathrm{~Hz}, \mathrm{H}-10^{\prime}$ ), 7.58 (s, $1 \mathrm{H}, \mathrm{H}-6$ ), 7.66 (brs, $1 \mathrm{H}, \mathrm{NH}), 7.70\left(\mathrm{dd}, 1 \mathrm{H},{ }^{3} \mathrm{~J}=7 \mathrm{~Hz},{ }^{4} \mathrm{~J}=2 \mathrm{~Hz}, \mathrm{H}-7^{\prime}\right), 7.73$ (dd, $\left.1 \mathrm{H},{ }^{3} \mathrm{~J}=6.9 \mathrm{~Hz},{ }^{4} \mathrm{~J}=2 \mathrm{~Hz}, \mathrm{H}-11^{\prime}\right)$.

3-\{[5-(4-Chlorophenyl)-2-furyl]methylene\}-5-(2-methoxy-5-methylphenyl)pyrrol-2(3H)-one (16e).Yield 58\%, m.p. $195-196{ }^{\circ} \mathrm{C}$ (ethanol). Anal. Calcd. for $\mathrm{C}_{23} \mathrm{H}_{18} \mathrm{ClNO}_{3}$ (391.8): C, 70.50; $\mathrm{H}, 4.63 ; \mathrm{Cl}, 9.05 ; \mathrm{N}, 3.57$. Found: $\mathrm{C}, 70.32 ; \mathrm{H}, 4.48 ; \mathrm{Cl}, 8.67 ; \mathrm{N}, 3.51 \% .{ }^{1} \mathrm{H}-\mathrm{NMR}\left(\mathrm{CDCl}_{3}\right) \delta_{\mathrm{H}}$ $2.33\left(\mathrm{~s}, 3 \mathrm{H}, 10-\mathrm{CH}_{3}\right), 4.05\left(\mathrm{~s}, 3 \mathrm{H}, 7-\mathrm{OCH}_{3}\right), 6.84\left(\mathrm{~d}, 1 \mathrm{H},{ }^{3} J=3.8 \mathrm{~Hz}, \mathrm{H}-3^{\prime}\right), 6.85\left(\mathrm{~d}, 1 \mathrm{H},{ }^{3} J=3.8\right.$ $\left.\mathrm{Hz}, \mathrm{H}-4^{\prime}\right), 6.90$ (d, $\left.1 \mathrm{H},{ }^{3} J=8.6 \mathrm{~Hz}, \mathrm{H}-8\right), 7.05$ (s, $\left.1 \mathrm{H}, \mathrm{H}-6\right), 7.19$ (dd, $1 \mathrm{H},{ }^{3} J=8.4 \mathrm{~Hz},{ }^{4} J=2 \mathrm{~Hz}$, H-9), 7.41 (dd, $\left.1 \mathrm{H},{ }^{3} J=6.5 \mathrm{~Hz},{ }^{4} J=1.9 \mathrm{~Hz}, \mathrm{H}-7^{\prime}\right), 7.44$ (dd, $\left.1 \mathrm{H},{ }^{3} J=6.5 \mathrm{~Hz},{ }^{4} J=2 \mathrm{~Hz}, \mathrm{H}-11^{\prime}\right)$, 7.58 (s, 1H, H-4), 7.60 (brs, $1 \mathrm{H}, \mathrm{NH}), 7.67\left(\mathrm{~d}, 1 \mathrm{H},{ }^{4} J=2 \mathrm{~Hz}, \mathrm{H}-6\right), 7.72\left(\mathrm{dd}, 1 \mathrm{H},{ }^{3} J=7 \mathrm{~Hz},{ }^{4} J=\right.$ $\left.2 \mathrm{~Hz}, \mathrm{H}-8^{\prime}\right), 7.75$ (dd, $\left.1 \mathrm{H},{ }^{3} J=6.9 \mathrm{~Hz},{ }^{4} J=2 \mathrm{~Hz}, \mathrm{H}-10^{\prime}\right)$.

\section{Acknowledgements}

This study was supported by the Grant Agency of the Slovak Ministry of Education (project Nos. $1 / 0069 / 03,1 / 0071 / 03$ and $1 / 8207 / 01$.

\section{References}

1. Gašparová, R.; Lácová, M. Collect. Czech. Chem. Commun. 1995, 60, 1178.

2. Lácová, M.; Gašparová, R.; Loos, D.; Liptay, T.; Prónayová, N. Molecules 2000, 5, 167.

3. Lácová, M.; Chovancová, J.; Veverková, E.; Toma, Š. Tetrahedron 1996, 52, 251.

4. Krutošíková, A.; Lácová, M.; Dandárová, M.; Chovancová J. ARKIVOC 2000, Vol. 1, (iii) Part 3, 409.

5. Galema, S. A. Chem. Soc. Rev. 1997, 26, 233.

6. Shindhar, D. R.; Sastry, C. V. R.; Vaidya, N. K. J. Indian. Chem. Soc. 1980, 57, 1118; Chem. Abstr. 1981, 94, 174765j.

7. Shigetaka, Y.; Akira, T.; Gang-Nan, Y.; Hsing-Chien, H. Yakugaku Zasshi 1970, 90, 1150; Chem. Abstr. 1970, 73, 120433x.

8. Konečný, V.; Krutošíková, A. Czech. 1978, 169, 549; Chem. Abstr. 1978, 88, 152397q.

9. Krutošíková, A.; Konečný V.; Kováć J. Collect. Czech. Chem. Commun. 1978, 43, 2643.

10. Deulofeu, V.; Guerrero, T. J. Org. Synth. Coll. 1955, 586. 
11. Cornthwaite, W. R.; Lazarus, S.; Snelling, R.H.; Denoon, C. E. J. Am. Chem. Soc. 1936, 58, 628.

12. Villemin, D.; Martin B. Synth. Commun. 1995, 25, 3135.

13. Villemin, D.; Ricard, M. Synth. Commun. 1987, 17, 283.

14. Perjéssy, A.; Toma, Š. Acta Fac. Rerum Natur. Univ. Comenianae, Chimia 1983, 31, 1.

15. Soulier, J. P.; Guegen J. Rev. Hemat. 1948, 3, 180.

16. Perjéssy, A.; Hrnčiar P. Spectrochim. Acta 1982, A 38, 499.

17. Boháč, A.; Perjessy, A.; Loos, D.; Hrnčiar, P. Monatsh. Chem. 1991, 122, 943.

18. Wolf, D. E.; Folkers, K. Org. Reaction 1951, 6, 449.

19. Fieser, L.F.; Fieser, M. Reagent For Organic Synthesis; John Wiley and Sons, Inc: New York, 1967; pp 402-403.

20. Lácová, M.; Hrnčiar, P. Collect. Czech. Chem. Commun. 1977, 42, 535.

21. Frimm, R.; Kováč, J.; Krutošíková, A. Chem. Zvesti 1973, 27, 101 and references in.

22. Royer, R.; Bisagni, E.; Menichi, G. Bull. Soc. Chim. France 1964, 9, 2112.

23. Lácová, M.; Volná, F. Acta Fac. Rerum Natur. Univ. Comenianae, Chimia 1972, 17, 1. 